\title{
colaboraciones
}

\section{Influencia del filler calizo en las propiedades de los morteros a resistencia constante}

\author{
FRANCISCO HERNÁNDEZ \\ ETSICCP UPM
}

IGNACIO MENÉNDEZ Y FERNANDO TRIVIÑO

ICCET/CSIC

ESPAÑA

Fecha de recepción: 24-IX-93

\section{RESUMEN}

En este artículo se estudian los efectos producidos por el "filler" calizo en el cemento portland al utilizar este cemento con adiciones, en la fabricación de morteros.

Se parte de un cemento portland al que se le añaden proporciones de "filler" calizo desde 0 hasta el 50\%, y se preparan probetas de mortero utilizando como árido arena normalizada, curándose a continuación hasta la edad de 28 dias, fecha en la que se someten a rotura por flexotracción y compresión.

La resistencia de los morteros se fija a la edad de 28 dias, haciéndola coincidir con la de un mortero de cemento patrón (cemento sin adiciones) a la misma edad, y se observan, a resistencia fija, los efectos del "filler" sobre el escurrimiento $y$ relación agua/cemento.

\section{$S U M M A R Y$}

This article studies the effects produced by the lime filler on the Portugal cement used with additions in the production of mortars.

The starting point is a Portland cement to which different ratios of lime filler, ranging from $0-50 \%$, are added. The next step consists of preparing mortar specimens using standarlized sand as aggregate, curing them up to the age of 28 days when they are put to flexo-tensile and compression tests.

The mortar strength is fixed at the age of 28 days, making it coincide with the strength of a pattern cement mortar (cement without additions) of the same age. Then the effects of the filler on the slump and the water cement relation are observed for fixed strength.

\section{INTRODUCCIÓN}

En la bibliografía se dan referencias (1) ...(14) de los efectos originados por la adición de "filler" calizo al cemento portland en proporciones, en la mayoría de los casos, no superiores al $15 \%$. Para mayores proporciones se tienen datos experimentales (3) (13) y (14) respecto a los tiempos de fraguado, resistencias y plasticidad del cemento y morteros.

Por lo general se han observado variaciones reológicas y mecánicas en función del porcentaje de "filler" calizo adicionado.
En España la industria cementera realizó ensayos previos con adiciones del $5 \%$ en "filler" (5).

La industria de la Construcción Francesa tiene datos prácticos para proporciones, de hasta el $20 \%$, ya que en Francia, Alemania y Hungría se admiten adiciones de "filler" en cementos portland superiores a las indicadas en el pliego RC-88 (15\%).

De acuerdo con los resultados que se obtuvieron (3) (13) (14) para proporciones de "filler" calizo de hasta 
el $50 \%$ podemos decir que se producen variaciones en el fraguado del cemento, en el escurrimiento de los morteros, y una tendencia a la caída de resistencias mecánicas, fundamentalmente para adiciones elevadas de "filler" calizo.

\section{PARTE EXPERIMENTAL}

\section{Preparación de la materia prima}

El cemento utilizado se obtiene a partir de un clínker molido y un yeso (sulfato cálcico hidratado R A: Merck), mezclados en proporciones tales que la relación final clínker/yeso sea de 9,34 (9,68\% en yeso), cantidad máxima tolerada según el pliego RC-88. La adición de yeso fue de $7,67 \%$, teniendo en cuenta que el contenido en $\mathrm{SO}_{3}$ del cemento portland es de $0,93 \%$ ( $2 \%$ en yeso).

En el análisis granulométrico del clínker, se comprobó que el $100 \%$ del tamaño de sus gránulos era inferior a $106 \mu \mathrm{m}$, y que el $93 \%$ lo era a $38 \mu \mathrm{m}$.

La caliza componente utilizada como adición se molió separadamente del clínker, presentando un problema de aglutinación lo que falsea la granulometría y, por consiguiente, el tamaño máximo de la muestra. Para evitar lo anteriormente descrito, se tamizó la muestra obtenida después de cada molienda repetidamente, haciéndola pasar por un tamiz de $30 \mu \mathrm{m}$ su totalidad, comprobando finalmente el tamaño máximo de la misma.

Hecho el análisis químico del clínker, se han obtenido los siguientes resultados:

$$
\begin{aligned}
& \text { Pérdida al fuego .............. } 0,39 \% \\
& \mathrm{Fe}_{2} \mathrm{O}_{3} \ldots \ldots \ldots \ldots \ldots \ldots \ldots \ldots \ldots . . . \ldots \ldots \ldots \\
& \hline
\end{aligned}
$$

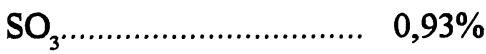

$$
\begin{aligned}
& \mathrm{SiO}_{3} \ldots \ldots \ldots \ldots \ldots \ldots \ldots \ldots . . \ldots \ldots \ldots \ldots \\
& \mathrm{CaO} \text {......................... 65,86\% } \\
& \mathrm{CaO} \text {.......... Libre ....... } 1,21 \% \\
& \mathrm{Al}_{2} \mathrm{O}_{3} \ldots \ldots \ldots \ldots \ldots \ldots \ldots \ldots . . .5,59 \% \\
& \mathrm{MgO} \ldots \ldots \ldots \ldots \ldots \ldots \ldots \ldots . . . . \ldots \ldots
\end{aligned}
$$

El cemento obtenido de la unión del clínker con el yeso se mezcló posteriormente con el "filler" calizo en las proporciones del $0,5,10,15,20,30,40$, y $50 \%$ formándose los respectivos cementos con adiciones los cuales se utilizarán posteriormente en este estudio.

Asimismo se realizó, sobre los morteros obtenidos, un estudio por difracción de rayos $\mathrm{X}$.

\section{Preparación de las muestras}

Se fabricó el mortero patrón empleando cemento portland con el $=\%$ de adiciones de "filler" calizo para una relación (w/c) agua/cemento de 0,50 (mortero normalizado); su resistencia media $\mathrm{R}_{\mathrm{m}}$ fue igual a $58 \pm 1$ $\mathrm{N} / \mathrm{mm}^{2}$. Asimismo se fabricaron los restantes morteros determinando, inicialmente, mediante cálculo y experiencias de laboratorio las relaciones agua/cemento y escurrimientos.

El mortero normalizado de cemento está constituido por 450 gramos de cemento, $1350 \mathrm{~g}$ de arena normalizada (UNE) y $225 \mathrm{~g}$ de agua. Asimismo los $450 \mathrm{~g}$ de cemento están constituidos por un núcleo de

\begin{tabular}{|c|c|c|c|c|c|c|c|}
\hline \multirow{3}{*}{$\begin{array}{c}\text { CLÍNKER } \\
+ \\
\text { YESO }\end{array}$} & \multirow{3}{*}{$\begin{array}{c}\text { FILLER } \\
\%\end{array}$} & \multirow{3}{*}{$\begin{array}{c}\text { RELACIÓN } \\
\text { Agua/Cemento } \\
(\mathbf{W} / \mathbf{C})\end{array}$} & \multirow{3}{*}{$\begin{array}{c}\text { Escurrimiento } \\
\%\end{array}$} & \multicolumn{4}{|c|}{ RESISTENCIA $\left(\mathbf{N} / \mathrm{mm}^{2}\right)$} \\
\hline & & & & \multicolumn{2}{|c|}{ Flexotracción } & \multicolumn{2}{|c|}{ Compresion } \\
\hline & & & & $\begin{array}{c}\text { Valor } \\
\text { parcial }\end{array}$ & $\begin{array}{l}\text { Valor } \\
\text { medio }\end{array}$ & $\begin{array}{c}\text { Valor } \\
\text { parcial }\end{array}$ & $\begin{array}{l}\text { Valor } \\
\text { medio }\end{array}$ \\
\hline 100 & 0 & 0,500 & $50 \pm 5$ & 7,5 & \multirow{3}{*}{7,5} & 58,6 & \multirow{3}{*}{58,5} \\
\hline 95 & 5 & 0,485 & $26 \pm 5$ & 7,7 & & 58,0 & \\
\hline 90 & 10 & 0,450 & $6 \pm 5$ & 7,2 & & 58,9 & \\
\hline 85 & 15 & 0,440 & 0 & \multicolumn{2}{|c|}{5,6} & \multicolumn{2}{|c|}{43,0} \\
\hline
\end{tabular}
clínker + yeso (en relación constante $=9,34)$ y "filler" calizo.

Las probetas utilizadas en los ensayos mecánicos son las normalizadas prismáticas de $4 \times 4 \times 16 \mathrm{~cm}$, que se curaron en cámara húmeda a $21 \pm 2{ }^{\circ} \mathrm{C}$ y $98 \%$ de hr.

Tabla I 


\section{Resultado de los ensayos}

Sólo se ha podido fabricar a resistencia constante morteros con proporciones de "filler" calizo de hasta el $10 \%$, ya que para valores del $15 \%$ y superiores no se consiguen las resistencias deseadas. Por otra parte el escurrimiento de los mismos es del $0 \%$, no pudiéndose realizar la compactación de los morteros. Por tales motivos sólo se han fabricado morteros con proporciones en "filler" calizo del 0, 5 y $10 \%$.

En la tabla I se dan los valores obtenidos para la relación agua/cemento, escurrimiento y resistencias a la edad de 28 días, a flexotracción y compresión.

\section{DRX}

Los trozos de probetas resultantes de las roturas a compresión, una vez triturados, se separa por tamizado la mayor parte de la arena del material cementante, realizándose sobre los mismos los correspondientes análisis por Difración de Rayos $\mathrm{X}$.

\section{Tratamiento de datos en DRX}

Se estudiaron los siguientes compuestos teniendo en cuenta los factores de reflexividad y las concentraciones de "filler" añadido:

$\begin{array}{llcc}\text { Compuestos } & \text { Fórmulas } & 2 \theta \text { ángulos } & \begin{array}{c}\text { Factor } \\ \text { reflexividad }\end{array} \\ \text { Sílice } & \mathrm{SiO}_{2} & 26,60 & 3,9 \\ \text { Calcita } & \mathrm{CaCO}_{3} & 29,40 & 2,0 \\ \text { Portlandita } & \mathrm{Ca}(\mathrm{OH})_{2} & 34,00 & 1,4 \\ \text { Yeso } & \mathrm{CaSO}_{4} \cdot 2 \mathrm{H}_{2} \mathrm{O} & 11,60 & 1,0 \\ \text { Ettringita } & \mathrm{Ca}_{7} \mathrm{Al}_{2} \mathrm{~S}_{3} \mathrm{O}_{3} \mathrm{O}_{2} \mathrm{H}_{2} \mathrm{O} & 9,11 & \\ \text { Alita } & \mathrm{Ca}_{3} \mathrm{SiO}_{3} & 51,80 & \end{array}$

Las cuentas obtenidas en dichos picos se dividieron entre los factores de reflexividad. Para compensar las adiciones de "filler" calizo y poder comparar la fracción clínker y llevar su contenido a 100 , se multiplicaron los resultados anteriores: portlandita, ettringita, yeso y alita por:

$$
\begin{aligned}
& 1,00 \text { para el } 0 \% \text { de "filler" } \\
& 1,07 \text { para el } 5 \% \text { de "filler" } \\
& 1,11 \text { para el } 10 \% \text { de "filler" } \\
& 1,18 \text { para el } 15 \% \text { de "filler" }
\end{aligned}
$$

Los resultados conseguidos figuran en la tabla II. Las distintas intensidades de cuarzo obtenidas por deficiencias en la separación árido silícico-cemento a través de la disgregación de las probetas y tamizado por el tamiz de 80 micras, están reflejadas en los resultados de cuarzo; éstos deberían haberse tenido en cuenta para compensar la fracción cementante que disminuye al aumentar el contenido en arena, pero prácticamente no influyeron en las cuentas del resto de los componentes.

\section{Discusión de resultados}

Sabemos por (3) (13) que hay una caída de resistencias a compresión al aumentar la cantidad añadida de carbonato cálcico, luego al fijar dicha resistencia es necesario variar las relaciones agua/cemento, lo cual incide sobre el escurrimiento de los morteros.

Al fijar la resistencia a compresión en $58 \pm 1 \mathrm{~N} / \mathrm{mm}^{2}$, a la edad de 28 días, la relación agua/cemento y el escurrimiento se hace cada vez menores a medida que aumenta el porcentaje de "fíller" calizo, ya que para mantener la resistencia es necesario reducir la relación

\begin{tabular}{|c|c|c|c|c|c|c|c|}
\hline \multicolumn{2}{|c|}{ CUENTAS $2 \theta$} & 26,60 & 29,40 & 34,00 & 9,11 & 32,60 & 11,63 \\
\hline $\begin{array}{l}\text { Filler } \\
\text { calizo }\end{array}$ & $\begin{array}{l}\text { Edad } \\
\text { (días) }\end{array}$ & $\mathrm{SiO}_{2}$ & $\mathrm{CaCO}_{3}$ & $\mathrm{Ca}(\mathrm{OH})_{2}$ & Ettringita & $\mathrm{C}_{3} \mathrm{~S}$ & Yeso \\
\hline 0 & 28 & 3.612 & 210 & 529 & 58 & 166 & 41 \\
\hline 5 & 28 & 2.663 & 317 & 520 & 72 & 114 & -- \\
\hline 10 & 28 & 5.256 & 317 & 490 & 84 & 104 & 41 \\
\hline 15 & 28 & 2.372 & 270 & 631 & 78 & -- & 63 \\
\hline
\end{tabular}
agua/cemento, y al reducir dicha relación decrece el escurrimiento. Para un porcentaje de "filler" calizo del

Tabla II 
$15 \%$ y una relación agua/cemento de 0,44 , el escurrimiento se hace cero y no se consigue la resistencia deseada (tabla I). Si queremos alcanzar la resistencia de $58 \pm 1 \mathrm{~N} / \mathrm{mm}^{2}$ con el $15 \%$ de "filler", sería necesario reducir el agua hasta alcanzar una relación agua/cemento de 0,33 , valor que nos imposibilita la compactación normal del mortero sin utilizar aditivos además de medios auxiliares. Por lo tanto, en igualdad de condiciones de compactación, sólo se han podido conseguir los objetivos planteados con adiciones de "filler" hasta el $10 \%$.

En la tabla II, sin tener en cuenta los datos de la sílice y utilizando los resultados DRX correspondientes al $\mathrm{Ca}(\mathrm{OH})_{2}$, ettringita, $\mathrm{C}_{3} \mathrm{~S}\left(3 \mathrm{CaOSiO}_{5}\right)$ y yeso, se observa que al aumentar la relación $\mathrm{CaCO}_{3} /$ clínker+yeso, tienden a mantenerse constantes los resultados del $\mathrm{Ca}(\mathrm{OH})_{2}$ y ettringita en la fase cemento. No se observa la formación de carboaluminatos.

La cantidad detectada de $\mathrm{C}_{3} \mathrm{~S}$ tiende a disminuir, y a partir del $15 \%$ no se aprecia.

\section{CONCLUSIONES}

a) Respecto a los observado en la fabricación de los morteros y de los valores de las resistencias mecánicas se infiere:

\section{Tabla I}

$1^{\circ} \mathrm{A}$ resistencia constante igual a la del mortero con cemento sin adiciones, sólo se pueden amasar morteros con porcentajes de "filler" calizo inferiores al $15 \%$. Para el $15 \%$ en "filler" la pérdida de resistencia es tal que, para mantenerla igual a la del mortero sin adiciones, es necesario reducir el agua hasta conseguir relaciones agua/cemento del orden de 0,31 a 0,33, lo que impide realizar el moldeo del mortero por métodos normales.

$2^{\circ}$ A resistencia constante, el escurrimiento se hace cero para adiciones de "filler" calizo en el cemento del orden del $15 \%$ dado que se necesita reducir, fuertemente, la relación agua/cemento con el fin de conseguir la resistencia prefijada.

$3^{\circ}$ A igual resistencia, las relaciones agua/cemento y los escurrimientos se hacen menores a medida que aumenta el porcentaje de "filler" en el cemento.

$4^{\circ}$ La resistencia fijada a 28 días no se alcanza con adiciones al cemento del $15 \%$.

\section{Tabla II}

b) Del estudio por DRX se infiere:

$1^{\circ}$ No ha tenido lugar la formación de carboaluminatos en ninguna de las muestras, muy posiblemente debido a que el yeso añadido no permite dicha formación favoreciendo la de la ettringita.

\section{AGRADECIMIENTO}

Los autores agradecen a la Dirección General de Investigación Científica y Técnica, el apoyo económico prestado para la elaboración de este proyecto PB 890189 , sin el cual no se podría haber realizado.

\section{BIBLIOGRAFÍA}

(1) "Limestone substitutes for gypsum as a cement ingredient" Bobros. Rock Products, pp 64/67. 1967.

(2) "Calcaire comme addition and cement". T. Gacesa y otros. Cement 21 (3-4) 107, 113, 1979.

(3) "The efect of calcareous filler on portland cement mortars". $\mathrm{M}^{\mathrm{a}} \mathrm{J}$. .Escorihuela, I. Menéndez, F. Triviño, F. Hernández, Hurtado, $M^{a}$ A. Matín.

(4) "Calcite as concrete and mixture". Elving Hognestad. Journal of the American Concrete Institute. Vol. $25 \mathrm{n}^{\circ} 9$ pp 801-803. 1954.

(5) "Conclusiones sobre Cementos de adicción". CDTI-OFICEMEN. Nov. 1980. 
(6) 'The effect of fillers on strength of Cement Mortars". I. Soroka and N.Setter. Cement and Concrete Research. Vol. 7 pp 449-456. 1977.

(7) "Calcareous filler and the compressive strength of Portland Cement". I. Soroka and N. Stern. Cement and concrete Resarch. Vol. 6, pp 367-376. 1976.

(8) "El punto sobre las nuevas normas decemento". Ciments et Chaux n 697. 1980.

(9) "Hydration of the Aluminous Minerals of Portland Cement in the presence of finely ground carbonate". P.P. Budnikov V.M Kolbasovs. Cement and Lime Manufacture. pp 60/61 G.Julio 1962.

(10) "Indagine sul differente comportamento degli inerti silicico a calcareo". Luissio Cassino. Giuseppe Pintor. Il cemento pp 255/262. 4/1972.

(11)"A review of limestons additions to portland cement and concrete". Kewin D. Ingranm Grand Kenneth E. Daugherty. Cement and Concrete Research 143 (1991), 165 - 17.

(12) "Influence des fillers sur les propiétes des mortiers et des betons". J. Bombled. Ciments. Beton. Plasters. Chau. $n^{0} 7385 / 82$.

(13) "Influencia de la adicción de "filler" calizo sobre el fraguado del cemento". I. Menéndez, F. Hernández, F. Triviño. Materiales de Construcción ICCET (CSIC) Madrid . Vol. 43-nº 231. julio/agosto/septiembre 1993.

\section{publicación del ICCET/CSIC}

\section{INSPECCION DE OBRAS DAÑADAS POR CORROSION DE ARMADURAS}

El presente Manual va dirigido principalmente a técnicos especializados y laboratorios que tienen que intervenir en el dictamen de la situación de deterioro de estructuras de hormigón armado dañadas por corrosión de armaduras.

Comienza con un resumen recordatorio de los factores principales a los que se pueden deber los daños prematuros por corrosión de armaduras, para seguir con algunas indicaciones de cómo se deben realizar las inspecciones, y de los ensayos y la metodologia que se recomienda realizar para poder dictaminar con precisión las causas de daño.

A continuación se hacen una serie de comentarios sobre la vida residual de estructuras dañadas, sobre el riesgo de corrosión futura, el seguimiento necesario de una estructura reparada y una breve enumeración de métodos de reparación y consideraciones básicas a tener en cuenta en la recomendación de un determinado método. Se aporta una breve relación bibliográfica.

Finalmente se incluyen en forma de ficha la descripción de algunos casos de corrosión de armaduras detectados en nuestro país.

\section{manual}

inspección de obras dañadas por corrosión

de armaduras 